\title{
ON AN ALGEBRA OF SIEGEL MODULAR FORMS ASSOCIATED WITH THE THETA GROUP $\Gamma_{2}(1,2)$
}

\author{
By
}

Takeyoshi Kogiso and Koji Tsushima

\begin{abstract}
In this note, we shall calculate some homogeneous polynomials explicitly based on B. Runge's conjecture in [4] p. 203 and give the explicit structure of the graded ring $A\left(\Gamma_{2}(1,2)\right)$ of Siegel modular forms of genus two belonging to the discrete subgroup $\Gamma_{2}(1,2)$ of $S p(2, R)$.
\end{abstract}

\section{§1. Notations and preliminaries}

Throughout this note, we will use the following notations.

We denote by $\boldsymbol{H}_{g}$ the Siegel upper half space of genus $g$ defined by

$$
\boldsymbol{H}_{g}:=\{Z \in M(g, C) \mid Z \text { : symmetric, } \operatorname{Im}(Z)>0\} \text {. }
$$

We denote by $S p(g, R)$ the usual real symplectic group of size $2 g$ defined by

$$
S p(g, \boldsymbol{R}):=\left\{\left.X \in M(2 g, \boldsymbol{R})\right|^{t} X J X=J, J=\left[\begin{array}{cc}
0 & I_{g} \\
-I_{g} & 0
\end{array}\right]\right\} .
$$

Furthermore we consider the following discrete subgroup of $S p(g, \boldsymbol{R})$,

$$
\Gamma_{g}:=S p(g, Z) \text {. }
$$

We call this $\Gamma_{g}$ the Siegel modular group; and we put

$$
\Gamma_{g}(n):=\left\{\left[\begin{array}{ll}
A & B \\
C & D
\end{array}\right] \in \Gamma_{g} \mid\left[\begin{array}{cc}
A & B \\
C & D
\end{array}\right] \equiv\left[\begin{array}{cc}
I_{g} & 0 \\
0 & I_{g}
\end{array}\right] \bmod n\right\}
$$

which is called the principal congruence subgroup of level $n$.

We put

$$
\Gamma_{g}(n, 2 n):=\left\{\left[\begin{array}{ll}
A & B \\
C & D
\end{array}\right] \in \Gamma_{g}(n) \mid\left(A^{t} B\right)_{0} \equiv\left(D^{t} C\right)_{0} \equiv 0 \bmod 2 n\right\}
$$



where for $M=\left(m_{i j}\right) \in M(g, C), M_{0}$ denotes the column vector $M_{0}=\left[\begin{array}{c}m_{11} \\ \vdots \\ m_{g g}\end{array}\right]$
of diagonal elements of $M$.

$\Gamma_{g}(1,2)$ is called the theta group.

The symplectic group $S p(g, R)$ acts transitively on the Siegel upper half space $\boldsymbol{H}_{g}$ by

$$
X(Z):=(A Z+B)(C Z+D)^{-1} \text { for } X=\left[\begin{array}{ll}
A & B \\
C & D
\end{array}\right] \in S p(g, \boldsymbol{R}), Z \in \boldsymbol{H}_{g} .
$$

We assume that $g \geq 2$.

For any non-negative integer $k$, we call $f(Z)$ a Siegel modular form of weight $k$ if

(i) $f$ is a holomorphic function on $\boldsymbol{H}_{g}$,

(ii) $f(X(Z))=\operatorname{det}(C Z+D)^{k} f(Z)$ for any $X=\left[\begin{array}{ll}A & B \\ C & D\end{array}\right] \in \Gamma_{g}$.

The space of all modular forms of the same weight $k$ has a structure of a vector space and we denote it by $\left[\Gamma_{g}, k\right]$. The direct sum of them becomes the finite generated graded algebra and we denote it by $A\left(\Gamma_{g}\right):=\oplus_{k}\left[\Gamma_{g}, k\right]$.

We use the classical notation for the thetas, i.e. for $(\tau, z) \in \boldsymbol{H}_{g} \times \boldsymbol{C}^{g}$,

$$
\theta\left[\begin{array}{l}
\alpha \\
\beta
\end{array}\right](\tau, z)=\sum_{x \in Z^{g}} \exp 2 \pi i\left(\frac{1}{2} \tau\left[x+\frac{1}{2} \alpha\right]+\left\langle x+\frac{1}{2} \alpha, z+\frac{1}{2} \beta\right\rangle\right)
$$

where $\langle x, y\rangle$ denotes the standard scalar product, $\tau[x]={ }^{t} x \tau x$ and $i$ denotes $\sqrt{-1}$.

The elements $\alpha$ and $\beta$ of $\boldsymbol{Z}^{g}$ are also regarded as elements in $\boldsymbol{F}_{2}^{g}$ with entries 0 and 1 . Here we remark that $\theta\left[\begin{array}{l}\alpha+2 p \\ \beta+2 q\end{array}\right]=\theta\left[\begin{array}{l}\alpha \\ \beta\end{array}\right](-1)^{\langle\alpha, q\rangle}$.

It is well known that $\theta\left[\begin{array}{l}\alpha \\ \beta\end{array}\right](\tau, z)$ is an analytic function on $\boldsymbol{H}_{g} \times \boldsymbol{C}^{g}$.

The $\theta\left[\begin{array}{l}\alpha \\ \beta\end{array}\right]=\theta\left[\begin{array}{l}\alpha \\ \beta\end{array}\right](\tau, 0)$ are called the theta constants.

In the case of $g=2$, there exists the following 10 even pairs of $\alpha, \beta$, i.e., $(-1)^{\langle\alpha, \beta\rangle}=1$ :

$$
\begin{aligned}
& \theta\left[\begin{array}{ll}
0 & 0 \\
0 & 0
\end{array}\right], \theta\left[\begin{array}{ll}
0 & 0 \\
0 & 1
\end{array}\right], \theta\left[\begin{array}{ll}
0 & 0 \\
1 & 0
\end{array}\right], \theta\left[\begin{array}{ll}
0 & 0 \\
1 & 1
\end{array}\right], \theta\left[\begin{array}{ll}
0 & 1 \\
0 & 0
\end{array}\right], \theta\left[\begin{array}{ll}
1 & 0 \\
0 & 0
\end{array}\right], \theta\left[\begin{array}{ll}
1 & 1 \\
0 & 0
\end{array}\right], \\
& \theta\left[\begin{array}{ll}
0 & 1 \\
1 & 0
\end{array}\right], \theta\left[\begin{array}{ll}
1 & 0 \\
0 & 1
\end{array}\right], \theta\left[\begin{array}{ll}
1 & 1 \\
1 & 1
\end{array}\right] .
\end{aligned}
$$


Now we define $\Theta:=\prod_{e v e n} \theta\left[\begin{array}{l}\alpha \\ \beta\end{array}\right]$. Here we note that the degree of $\Theta$ is 10 .

We use this $\Theta$ to describe our problem (posed by B. Runge).

Finally we have to prepare the definition of a code. A code $C$ means a linear subspace $C$ of $\boldsymbol{F}_{2}^{n}$, when its dimension $k$ and the minimal weight $d$, it is denoted by $[n, k, d]$. Where the weight $d$ means the number of entries 1 in an element $\alpha$ of a code $C$. A code is called doubly-even if the weight of every element of the code is divisible by 4 . A code $C$ is called the self-dual when it coinsides with its dual $C^{\perp}=\left\{x \in F_{2}^{n} \mid\langle x, y\rangle=0\right.$ for any $\left.y \in C\right\}$, where $\langle x, y\rangle$ means the standard inner product.

§. The relation between the Siegel modular group $\Gamma_{g}$ and the corresponding finite group

We can choose the following generator of $\Gamma_{g}$ :

$$
\Gamma_{g}=\left\langle\left[\begin{array}{cc}
I_{g} & S \\
0 & I_{g}
\end{array}\right],\left[\begin{array}{cc}
0 & I_{g} \\
-I_{g} & 0
\end{array}\right],{ }^{t} S=S \in M(g, Z)\right\rangle .
$$

On the other hand, the thetas of second order are given by

$$
f_{a}(\tau):=\theta\left[\begin{array}{l}
a \\
0
\end{array}\right](2 \tau)=\sum_{x \in \boldsymbol{Z}^{g}} \exp 2 \pi i\left(\tau\left[x+\frac{1}{2} a\right]\right)
$$

for $a \in Z^{g}$, where $i$ denotes $\sqrt{-1}$.

The function $f_{a}$ depends only on $a \bmod 2$, here $a$ is considered also as the element of $\boldsymbol{F}_{2}^{g}$.

The action of $\Gamma_{g}$ on thetas of second order $f_{a}=\theta\left[\begin{array}{l}a \\ 0\end{array}\right](2 \tau, 0)$ is the following.

Proposition 2.1 (see [3], p. 60). (1) For $\sigma=\left[\begin{array}{cc}I_{g} & S \\ 0 & I_{g}\end{array}\right] \in \Gamma_{g}, \sigma\left(f_{a}\right)=i^{S[a]} f_{a}$ with $S[a]={ }^{t} a S a$.

(2) We define the $2^{g} \times 2^{g}$-matrix $T_{g}$ by $T_{g}:=\left(\frac{1+i}{2}\right)^{g}\left((-1)^{\langle a, b\rangle}\right)_{a, b} \in \boldsymbol{F}_{2}^{g}$.

Then $J=\left[\begin{array}{cc}0 & I_{g} \\ -I_{g} & 0\end{array}\right]$ acts on $f_{a}$ as follows:

$$
J\left(f_{a}\right)=\sqrt{\operatorname{det}(-\tau)} \sum_{b \in F_{2}^{g}}\left(T_{g}\right)_{a, b} f_{a}
$$

for $f_{a}=\theta\left[\begin{array}{l}a \\ 0\end{array}\right](2 \tau, 0)$. 
Then we can consider the following $2^{g} \times 2^{g}$-matrix $D_{S}$ :

$D_{S}=\operatorname{diag}\left(i_{i}[a]\right)$ for $a \in F_{2}^{g}$, and we define $H_{g}:=\left\langle T_{g}, D_{S}\right\rangle$.

Here $H_{g}$ acts faithfully on the vector space generated by $f_{a}, a \in \boldsymbol{F}_{2}^{g}$ and $H_{g} /\{ \pm 1\}$ acts on the algebra $C\left[f_{a} f_{b}\right]_{a, b \in F_{2}^{g}}$.

Then the following theorem is known.

Proposition 2.2 (see [3], p. 60). There exists the surjective group homomorphism $\phi: \Gamma_{g} \rightarrow H_{g} /\{ \pm 1\}$ satisfying $\phi(J)=T_{g}$ and $\phi(\sigma)=\sigma\left(\left[\begin{array}{cc}I_{g} & S \\ 0 & I_{g}\end{array}\right]\right)=$ $D_{S}$ with the notations in proposition 2.1 .

By the surjective group homomorphism $\phi$ in above proposition 2.2 , the subgroup $\Gamma_{2}(1,2)$ of $\Gamma_{2}$ corresponds to the following finite group $G$ :

$$
G=\left\langle S_{4}, \operatorname{diag}( \pm 1, \pm 1, \pm 1, \pm 1), M_{1}=\left(\frac{1+i}{2}\right)\left[\begin{array}{cc}
I_{2} & I_{2} \\
I_{2} & -I_{2}
\end{array}\right]\right\rangle
$$

where $S_{4}$ denotes the symmetric group of degree 4 .

\section{§3. Runge's conjecture}

From above proposition 2.1 and proposition $2.2 \S 2$, B. Runge proved the following theorem 3.1 about the correspondence between the ring of modular forms and the invariant ring of some finite group by using the theta functions (see [3]).

THEOREM 3.1 (B. Runge). For any element $a=\left(a_{1}, \ldots, a_{g}\right)$ in $F_{2}^{g}$, we define the function

$$
Y_{a}: \overbrace{F_{2}^{n} \times \cdots \times F_{2}^{n}}^{g} \rightarrow Z_{+}
$$

by

$$
Y_{a}\left(\gamma_{1}, \ldots, \gamma_{g}\right):=\#\left\{i \mid a=\left(\gamma_{i, 1}, \ldots, \gamma_{i, g}\right)\right\}
$$

where

$$
\left(\gamma_{1}, \ldots, \gamma_{g}\right)=\left[\begin{array}{ccc}
\gamma_{11} & \cdots & \gamma_{1 g} \\
\vdots & & \vdots \\
\gamma_{n 1} & \cdots & \gamma_{n g}
\end{array}\right] .
$$

Then for a self-dual doubly-even code $C$, the polynomial of $2^{g}$-variables 


$$
P_{g}(C):=\sum_{\left(\gamma_{1}, \ldots, \gamma_{g}\right) \in C^{g}} \prod_{a \in \boldsymbol{F}_{2}^{g}} f_{a}^{Y_{a}\left(\gamma_{1}, \ldots, \gamma_{g}\right)}
$$

with the thetas of second order

$$
f_{a}=\theta\left[\begin{array}{l}
a \\
0
\end{array}\right](2 \tau, 0)
$$

becomes a modular form (for the full modular group) of weight $k=n / 2$.

From the fact in [1], p. 177, the fact in [2], theorem 7.1 in p. 200, the statement of p. 201 and Lemma 2.1 of p. 180 in [4], the space generated by the polynomials $P_{2}(C)$ for $C=C^{\perp}$ becomes the invariant ring $C\left[f_{a} ; a \in F_{2}^{2}\right]^{G^{\prime}}$ for $G^{\prime}=\left\langle S_{4}, \operatorname{diag}( \pm 1, \pm 1, \pm 1, \pm 1), W=1 / \sqrt{2}\left[\begin{array}{cc}I_{2} & I_{2} \\ I_{2} & -I_{2}\end{array}\right]\right\rangle$, where the matrix $M_{1}$ of $G=\phi\left(\Gamma_{2}(1,2)\right)$ and $W$ of $G^{\prime}$ differ by an eight root of unity. Furthermore, from the arguement in p. 202, p. 203 in [4], for our case of $G=\phi\left(\Gamma_{2}(1,2)\right)$, $A\left(\Gamma_{2}(1,2)\right)=\oplus_{k}\left[\Gamma_{2}(1,2), k\right]$ corresponds to a certain subring of $C\left[f_{a}, a \in \boldsymbol{F}_{2}^{2}, \Theta\right]$ where $\Theta=\Pi_{\text {even }} \theta\left[\begin{array}{l}\alpha \\ \beta\end{array}\right]$.

Here, let us recall the related facts from the invariant theory.

Proposition 3.2 (in [3], p. 76). Let $G$ be a finite group acting on a polynomial ring $A=C\left[X_{1}, \ldots, X_{r}\right]$ and if $R=A^{G}$ is the invariant ring, it is known that $R$ is of the type:

$$
\boldsymbol{R}=\boldsymbol{C}\left[g_{1}, \ldots, g_{r}\right] \oplus \boldsymbol{C}\left[g_{1}, \ldots, g_{r}\right] g_{r+1} \oplus \cdots \oplus \boldsymbol{C}\left[g_{1}, \ldots, g_{r}\right] g_{n} \quad(r \leq n)
$$

where each $g_{i}$ is a homogeneous polynomial of degree $d_{i}$.

We denote by $R_{(l)}$ the homogeneous part of degree $l$ in $R$. Then we have

$$
\Phi_{G}(\lambda)=\sum_{l \geq 0} \operatorname{dim}_{C} R_{(l)} \lambda^{l}=\frac{1+\sum_{i=r+1, \ldots, n} \lambda^{d_{i}}}{\prod_{i=1, \ldots, r}\left(1-\lambda^{d_{i}}\right)} .
$$

In [4], p. 203, B. Runge calculated $\Phi_{\phi\left(\Gamma_{2}(1,2)\right)}(\sqrt{\lambda})$ as follows:

$$
\Phi_{\phi\left(\Gamma_{2}(1,2)\right)}(\sqrt{\lambda})=\frac{1+\lambda^{6}+\lambda^{8}+\lambda^{10}+\lambda^{19}+\lambda^{21}+\lambda^{23}+\lambda^{29}}{\left(1-\lambda^{4}\right)^{2}\left(1-\lambda^{6}\right)\left(1-\lambda^{12}\right)}
$$

and he remarked that this Poincaré series accords with [2], p. 405. Then he described the following conjecture: 
Conjecture (Runge) (in [4], p. 203). The ring $\oplus_{k}\left[\Gamma_{2}(1,2), k\right]$ has the following graded ring structure.

$$
\begin{aligned}
\oplus_{k}\left[\Gamma_{2}(1,2), k\right]= & C\left[P_{2}^{4}, P_{2}^{3} P_{6}, P_{2}^{2} P_{6}^{2}, P_{2} P_{6}^{3}, P_{6}^{4}, P_{8}, P_{12}, P_{28} \Theta, P_{32} \Theta, P_{36} \Theta\right] \\
= & C\left[P_{2}^{4}, P_{6}^{4}, P_{8}, P_{12}\right] \\
& \oplus C\left[P_{2}^{4}, P_{6}^{4}, P_{8}, P_{12}\right] P_{2}^{3} P_{6} \\
& \oplus C\left[P_{2}^{4}, P_{6}^{4}, P_{8}, P_{12}\right] P_{2}^{2} P_{6}^{2} \\
& \oplus C\left[P_{2}^{4}, P_{6}^{4}, P_{8}, P_{12}\right] P_{2} P_{6}^{3} \\
& \oplus C\left[P_{2}^{4}, P_{6}^{4}, P_{8}, P_{12}\right] P_{28} \Theta \\
& \oplus C\left[P_{2}^{4}, P_{6}^{4}, P_{8}, P_{12}\right] P_{32} \Theta \\
& \oplus C\left[P_{2}^{4}, P_{6}^{4}, P_{8}, P_{12}\right] P_{36} \Theta \\
& \oplus C\left[P_{2}^{4}, P_{6}^{4}, P_{8}, P_{12}\right] Q_{58}
\end{aligned}
$$

where $\Theta=\Pi_{\text {even }} \theta\left[\begin{array}{l}\alpha \\ \beta\end{array}\right], P_{i}$ is a certain homogeneous polynomial of degree $i$ and $Q_{58}$ is a certain polynomial of degree 58, product of such $P_{i}$ 's and $\Theta$.

In this note, we give an affirmative answer about the conjecture.

Actually B. Runge showed in the case of even weight related to this conjecture that:

THEOREM 3.3 (Runge) (see [4], p. 202). The ring $\oplus_{2 \mid k}\left[\Gamma_{2}(1,2), k\right]$ has the following graded ring structure.

$$
\begin{aligned}
\oplus_{2 \mid k}\left[\Gamma_{2}(1,2), k\right]= & C\left[P_{2}^{4}, P_{2}^{3} P_{6}, P_{2}^{2} P_{6}^{2}, P_{2} P_{6}^{3}, P_{6}^{4}, P_{8}, P_{12}\right] \\
= & C\left[P_{2}^{4}, P_{6}^{4}, P_{8}, P_{12}\right] \\
& \oplus C\left[P_{2}^{4}, P_{6}^{4}, P_{8}, P_{12}\right] P_{2}^{3} P_{6} \\
& \oplus C\left[P_{2}^{4}, P_{6}^{4}, P_{8}, P_{12}\right] P_{2}^{2} P_{6}^{2} \\
& \oplus C\left[P_{2}^{4}, P_{6}^{4}, P_{8}, P_{12}\right] P_{2} P_{6}^{3}
\end{aligned}
$$


where each $P_{i}$ are the following polynomials:

$$
\begin{aligned}
& P_{2}=(2), \\
& P_{6}=(6)-5(4,2)+30(2,2,2), \\
& P_{8}=(8)+14(4,4)+168(2,2,2,2),
\end{aligned}
$$

and

$$
P_{12}=(12)-33(8,4)+330(4,4,4)+792(6,2,2,2)
$$

where

$$
\left(a_{1}, a_{2}, a_{3}, a_{4}\right):=\sum_{\sigma \in S_{4} / \operatorname{Stab}\left(a_{1}, a_{2}, a_{3}, a_{4}\right)} \prod_{i=1, \ldots, 4}\left(f_{i-1}^{a_{i}}\right)^{\sigma} .
$$

Here we put $f_{0}=f_{[0]}^{0}, f_{1}=f_{\left[\begin{array}{l}0 \\ 1\end{array}\right]}, f_{2}=f_{\left[\begin{array}{l}{[0} \\ 0\end{array}\right]}, f_{3}=f_{[1]}$.

In this case, the corresponding finite group $G=\phi\left(\Gamma_{2}(1,2)\right)$ is given as follows.

$$
G=\left\langle S_{4},\left[\begin{array}{llll} 
\pm 1 & & & \\
& \pm 1 & & \\
& & \pm 1 & \\
& & & \pm 1
\end{array}\right], M_{1}=\frac{1+i}{2}\left[\begin{array}{cccc}
1 & 0 & 1 & 0 \\
0 & 1 & 0 & 1 \\
1 & 0 & -1 & 0 \\
0 & 1 & 0 & -1
\end{array}\right]\right\rangle \subset G L(4, C) .
$$

By theorem 3.3, what we have to consider are homogeneous polynomials of odd weight.

\section{§4. The calculations and the result}

By the invariance of the action of $S_{4}$ and $\operatorname{diag}( \pm 1, \pm 1, \pm 1, \pm 1)$, we may put

$$
\begin{aligned}
& P_{28}=\sum_{\substack{a_{1}+a_{2}+a_{3}+a_{4}=28 \\
a_{1}, a_{2}, a_{3}, a_{4} \text { are different odd integers }}} C_{a_{1}, a_{2}, a_{3}, a_{4}}\left(a_{1}, a_{2}, a_{3}, a_{4}\right)^{A}, \\
& P_{32}=\sum_{\substack{a_{1}+a_{2}+a_{3}+a_{4}=32 \\
a_{1}, a_{2}, a_{3}, a_{4} \text { are different odd integers }}} C_{a_{1}, a_{2}, a_{3}, a_{4}\left(a_{1}, a_{2}, a_{3}, a_{4}\right)^{A}}
\end{aligned}
$$

and

$$
P_{36}=\sum_{\substack{a_{1}+a_{2}+a_{3}+a_{4}=36 \\ a_{1}, a_{2}, a_{3}, a_{4} \text { are different odd integers }}} C_{a_{1}, a_{2}, a_{3}, a_{4}}\left(a_{1}, a_{2}, a_{3}, a_{4}\right)^{A}
$$


where $C_{a_{1}, a_{2}, a_{3}, a_{4}}$ 's are the constants of rational integers and

$$
\left(a_{1}, a_{2}, a_{3}, a_{4}\right)^{A}:=\sum_{\sigma \in S_{4}} \operatorname{sign}(\sigma) \prod_{i=1, \ldots, 4}\left(f_{i-1}^{a_{i}}\right) \sigma .
$$

By the invariance of the action of $M_{1}$, we calculate using the computer, nonzero alternating polynomials $P_{28}, P_{32}$ and $P_{36}$ as follows:

$$
\begin{gathered}
P_{28}=(19,5,3,1)^{A}-3(17,7,3,1)^{A}+(15,9,3,1)^{A} \\
-6(15,7,5,1)^{A}+5(13,11,3,1)^{A}+16(13,9,5,1)^{A} \\
-54(13,7,5,3)^{A}+13(11,9,7,1)^{A}-39(11,9,5,3)^{A}, \\
P_{32}=(21,7,3,1)^{A}-7(19,9,3,1)^{A}+21(17,11,3,1)^{A}-63(17,7,5,3)^{A} \\
-35(15,13,3,1)^{A}-18(15,9,7,1)^{A}+203(15,9,5,3)^{A}+63(13,11,7,1)^{A} \\
-294(13,11,5,3)^{A}-322(13,9,7,3)^{A}+2457(11,9,7,5)^{A}
\end{gathered}
$$

and

$$
\begin{aligned}
P_{36}= & 15(25,7,3,1)^{A}-75(23,9,3,1)^{A}+46(23,7,5,1)^{A}+120(21,11,3,1)^{A} \\
& -276(21,9,5,1)^{A}-1074(21,7,5,3)^{A}+644(19,13,3,1)^{A}-135(19,9,7,1)^{A} \\
& +1610(19,9,5,3)^{A}-210(17,15,3,1)^{A}-644(17,13,5,1)^{A} \\
& +266(17,11,5,3)^{A}-1665(17,9,7,3)^{A}+1254(15,13,7,1)^{A} \\
& -1330(15,13,5,3)^{A}+1956(15,11,9,1)^{A}-8736(15,11,7,3)^{A} \\
& +34690(15,9,7,5)^{A}-15750(13,11,9,3)^{A}+20930(13,11,7,5)^{A} .
\end{aligned}
$$

So we have $\oplus_{k}\left[\Gamma_{2}(1,2), k\right]$ contains $C\left[P_{2}^{4}, P_{2}^{3} P_{6}, P_{2}^{2} P_{6}^{2}, P_{2} P_{6}^{3}, P_{6}^{4}, P_{8}, P_{12}\right.$, $\left.P_{28} \Theta, P_{32} \Theta, P_{36} \Theta\right]$ as a subring.

Furthermore we remark that

$$
\begin{aligned}
\Phi_{\phi\left(\Gamma_{2}(1,2)\right)}(\sqrt{\lambda})= & \frac{1+\lambda^{6}+\lambda^{8}+\lambda^{10}+\lambda^{19}+\lambda^{21}+\lambda^{23}+\lambda^{29}}{\left(1-\lambda^{4}\right)^{2}\left(1-\lambda^{6}\right)\left(1-\lambda^{12}\right)} \\
= & 1+2 \lambda^{4}+2 \lambda^{6}+4 \lambda^{8}+5 \lambda^{10}+9 \lambda^{12}+9 \lambda^{14}+15 \lambda^{16}+17 \lambda^{18}+\lambda^{19}+23 \lambda^{20} \\
& +\lambda^{21}+27 \lambda^{22}+3 \lambda^{23}+36 \lambda^{24}+3 \lambda^{25}+39 \lambda^{26}+6 \lambda^{27}+7 \lambda^{29}+\ldots \ldots,
\end{aligned}
$$


and we checked that

$$
\begin{aligned}
& Q_{48}:=P_{2}^{3} P_{6} P_{36}=15(37,7,3,1)^{A}-105(35,9,3,1)^{A}+16(35,7,5,1)^{A} \\
& +15(33,11,3,1)^{A}-128(33,9,5,1)^{A}-821(33,7,5,3)^{A} \\
& +615(31,13,3,1)^{A}+144(31,11,5,1)^{A}+3590(31,9,7,1)^{A} \\
& +5681(31,9,5,3)^{A}-405(29,15,3,1)^{A}+512(29,13,5,1)^{A} \\
& -2633(29,11,7,1)^{A}+7258(29,11,5,3)^{A}-21320(29,9,7,3)^{A} \\
& -1485(27,17,3,1)^{A}-944(27,15,5,1)^{A}+103(27,13,7,1)^{A} \\
& +182(27,13,5,3)^{A}-31106(27,11,9,1)^{A}-111872(27,11,7,3)^{A} \\
& -68689(27,9,7,5)^{A}+1275(25,19,3,1)^{A}-640(25,17,5,1)^{A} \\
& +18826(25,15,7,1)^{A}+377(25,15,5,3)^{A}-12800(25,13,9,1)^{A} \\
& +88000(25,13,7,3)^{A}-136786(25,11,9,3)^{A}-104221(25,11,9,5)^{A} \\
& +1875(23,21,3,1)^{A}+2000(23,19,5,1)^{A}-24412(23,17,7,1)^{A} \\
& -29837(23,17,5,3)^{A}+11122(23,15,9,1)^{A}+346624(23,15,7,3)^{A} \\
& +57819(23,13,11,1)^{A}+207430(23,13,9,3)^{A}-296200(23,15,7,3)^{A} \\
& +688701(23,11,9,5)^{A}-36913(21,19,7,1)^{A}-30788(21,19,5,3)^{A} \\
& -85376(21,17,9,1)^{A}+82478(21,17,7,3)^{A}+178738(21,15,11,1)^{A} \\
& +209085(21,15,9,3)^{A}-1453960(21,15,7,5)^{A}-417746(21,13,11,3)^{A} \\
& +243712(21,13,9,5)^{A}+4563377(21,11,9,7)^{A}+92575(19,17,11,1)^{A} \\
& -108025(19,17,9,3)^{A}-1194129(19,17,7,5)^{A}+230501(19,15,13,1)^{A} \\
& -874752(19,15,11,3)^{A}-1942435(19,15,9,5)^{A}-2084558(19,13,11,5)^{A} \\
& +8801090(19,13,9,7)^{A}-319143(17,15,13,3)^{A}-2492914(17,15,11,5)^{A} \\
& +4411534(17,15,9,7)^{A}+4772515(17,13,11,7)^{A}+1161508(15,13,11,9)^{A}
\end{aligned}
$$

is different from any linear combinations of the following polynomials: 


$$
\begin{aligned}
& P_{2}^{4} P_{12} P_{28}=(39,5,3,1)^{A}+(37,7,3,1)^{A}-38(35,9,3,1)^{A}+813(35,7,5,1)^{A} \\
& -38(33,11,3,1)^{A}+760(33,9,5,1)^{A}+733(33,7,5,3)^{A} \\
& +142(31,13,3,1)^{A}+\ldots \ldots \\
& P_{8} P_{12} P_{28}=(39,5,3,1)^{A}-3(37,7,3,1)^{A}-18(35,9,3,1)^{A}+805(35,7,5,1)^{A} \\
& +62(33,11,3,1)^{A}-2360(33,9,5,1)^{A}-2319(33,7,5,3)^{A} \\
& -518(31,13,3,1)^{A}+\ldots \ldots \\
& P_{8} P_{2}^{4} P_{32}=(37,7,3,1)^{A}-3(35,9,3,1)^{A}+4(35,7,5,1)^{A}+13(33,11,3,1)^{A} \\
& -16(33,9,5,1)^{A}-71(33,7,5,3)^{A}-31(31,13,3,1)^{A}+\ldots \ldots, \\
& P_{8}^{2} P_{32}=(37,7,3,1)^{A}-7(35,9,3,1)^{A}+49(33,11,3,1)^{A}-63(33,7,5,3)^{A} \\
& -231(31,13,3,1)^{A}+28(31,13,3,1)^{A}+\ldots \ldots \\
& P_{2}^{8} P_{32}=(37,7,3,1)^{A}+(35,9,3,1)^{A}-7(33,11,3,1)^{A}+56(33,9,5,1)^{A} \\
& -63(33,7,5,3)^{A}-596(31,13,3,1)^{A}+\ldots \ldots,
\end{aligned}
$$

and

$$
\begin{aligned}
P_{12} P_{36}= & 15(37,7,3,1)^{A}-75(35,9,3,1)^{A}+46(35,7,5,1)^{A}-375(33,11,3,1)^{A} \\
& -276(33,9,5,1)^{A}-1074(33,7,5,3)^{A}+2475(31,13,3,1)^{A}+\ldots \ldots
\end{aligned}
$$

Furthermore we get these 7 polynomials $\times \Theta$ as a basis of the 7-dimensional vector space $R_{29}$, i.e.

$$
\begin{aligned}
R_{29}=\langle & Q_{48} \Theta=P_{2}^{3} P_{6} P_{36} \Theta, P_{2}^{4} P_{12} P_{28} \Theta, P_{8} P_{12} P_{28} \Theta, P_{8} P_{2}^{4} P_{32} \Theta \\
& \left.P_{8}^{2} P_{32} \Theta, P_{2}^{8} P_{32} \Theta, P_{12} P_{36} \Theta\right\rangle
\end{aligned}
$$

(We checked that $P_{2}^{2} P_{6}^{2} P_{32} \Theta$ and $P_{2} P_{6}^{3} P_{28} \Theta$ are linear combinations of above 7 polynomials $\times \Theta$.)

So we have the following decomposition: 


$$
\begin{aligned}
& C\left[P_{2}^{4}, P_{2}^{3} P_{6}, P_{2}^{2} P_{6}^{2}, P_{2} P_{6}^{3}, P_{6}^{4}, P_{8}, P_{12}, P_{28} \Theta, P_{32} \Theta, P_{36} \Theta\right] \\
&= C\left[P_{2}^{4}, P_{6}^{4}, P_{8}, P_{12}\right] \\
& \oplus C\left[P_{2}^{4}, P_{6}^{4}, P_{8}, P_{12}\right] P_{2}^{3} P_{6} \\
& \oplus C\left[P_{2}^{4}, P_{6}^{4}, P_{8}, P_{12}\right] P_{2}^{2} P_{6}^{2} \\
& \oplus C\left[P_{2}^{4}, P_{6}^{4}, P_{8}, P_{12}\right] P_{2} P_{6}^{3} \\
& \oplus C\left[P_{2}^{4}, P_{6}^{4}, P_{8}, P_{12}\right] P_{28} \Theta \\
& \oplus C\left[P_{2}^{4}, P_{6}^{4}, P_{8}, P_{12}\right] P_{32} \Theta \\
& \oplus C\left[P_{2}^{4}, P_{6}^{4}, P_{8}, P_{12}\right] P_{36} \Theta \\
& \oplus C\left[P_{2}^{4}, P_{6}^{4}, P_{8}, P_{12}\right] Q_{48} \Theta \\
& \oplus \ldots
\end{aligned}
$$

and using this result, we see that the Poincaré series of $\oplus_{k}\left[\Gamma_{2}(1,2), k\right]$ and that of this subring coinside. This implies that

$$
\begin{aligned}
\oplus_{k}\left[\Gamma_{2}(1,2), k\right]= & C\left[P_{2}^{4}, P_{2}^{3} P_{6}, P_{2}^{2} P_{6}^{2}, P_{2} P_{6}^{3}, P_{6}^{4}, P_{8}, P_{12}, P_{28} \Theta, P_{32} \Theta, P_{36} \Theta\right] \\
= & C\left[P_{2}^{4}, P_{6}^{4}, P_{8}, P_{12}\right] \\
& \oplus C\left[P_{2}^{4}, P_{6}^{4}, P_{8}, P_{12}\right] P_{2}^{3} P_{6} \\
& \oplus C\left[P_{2}^{4}, P_{6}^{4}, P_{8}, P_{12}\right] P_{2}^{2} P_{6}^{2} \\
& \oplus C\left[P_{2}^{4}, P_{6}^{4}, P_{8}, P_{12}\right] P_{2} P_{6}^{3} \\
& \oplus C\left[P_{2}^{4}, P_{6}^{4}, P_{8}, P_{12}\right] P_{28} \Theta \\
& \oplus C\left[P_{2}^{4}, P_{6}^{4}, P_{8}, P_{12}\right] P_{32} \Theta \\
& \oplus C\left[P_{2}^{4}, P_{6}^{4}, P_{8}, P_{12}\right] P_{36} \Theta \\
& \oplus C\left[P_{2}^{4}, P_{6}^{4}, P_{8}, P_{12}\right] P_{2}^{3} P_{6} P_{36} \Theta .
\end{aligned}
$$

\section{Acknowledgement}

The authors are grateful to Prof. B. Runge for suggesting this work and to Prof. T. Kimura for discussing about this work. 


\section{References}

[1] E. Bannai, A survey of the invariant rings of finite groups and automorphic forms, (in Japnese) Report on symposium of algebra held at Yamagata (1996), 173-187.

[2] J-I Igusa, On Siegel modular forms of genus two (II), Am. J. Math. 86 (1964), 392-412.

[ 3 ] B. Runge, On Siegel modular forms, Part I, J. reine. angew. Math 436 (1993), 57-85.

[4] — Codes and Siegel modular forms, Discrete Math. 148 (1996), 175-204.

Institute of Mathematics

University of Tsukuba

Ibaraki, 305, Japan 